\title{
IRAS 15099-5856: REMARKABLE MID-INFRARED SOURCE WITH PROMINENT CRYSTALLINE SILICATE EMISSION EMBEDDED IN THE SUPERNOVA REMNANT MSH15-52
}

\author{
Bon-Chul Koo ${ }^{1}$, Christopher F. McKee ${ }^{2,3}$, Kyung-Won SuH ${ }^{4}$, Dae-Sik Moon ${ }^{5}$, Takashi Onaka ${ }^{6}$, Michael G. Burton ${ }^{7}$, \\ Masaaki Hiramatsu ${ }^{8}$, Michael S. Bessell ${ }^{9}$, B. M. Gaensler ${ }^{10}$, Hyun-Jeong Kim ${ }^{1}$, Jae-Joon LeE ${ }^{11,12}$, \\ Woong-Seob JeOng $^{12}$, Ho-Gyu LeE ${ }^{5}$, Myungshin Im ${ }^{1}$, Ken'ichi Tatematsu ${ }^{13}$, Kotaro Kohno ${ }^{14}$, Ryohei Kawabe ${ }^{13}$, \\ Hajime Ezawa ${ }^{13}$, Grant Wilson ${ }^{15}$, Min S. Yun ${ }^{15}$, and David H. Hughes ${ }^{16}$ \\ ${ }^{1}$ Department of Physics and Astronomy, Seoul National University, Seoul 151-742, Republic of Korea; koo@ astrohi.snu.ac.kr \\ ${ }^{2}$ Department of Physics and Astronomy, University of California, Berkeley, CA 94720, USA \\ ${ }^{3}$ Laboratoire d'Etudes du Rayonnement et de la Matière en Astrophysique, LERMA-LRA, Ecole Normale Supérieure, 24 rue Lhomond, 75005 Paris, France \\ ${ }^{4}$ Department of Astronomy and Space Science, Chungbuk National University, Cheingju-City 361-763, Republic of Korea \\ ${ }^{5}$ Department of Astronomy \& Astrophysics, University of Toronto, Toronto, ON M5S 3H4, Canada \\ ${ }^{6}$ Department of Astronomy, University of Tokyo, Bunkyo-ku, Tokyo 113-0033, Japan \\ ${ }^{7}$ School of Physics, University of New South Wales, Sydney, New South Wales 2052, Australia \\ ${ }^{8}$ Academia Sinica Institute of Astronomy and Astrophysics, P.O. Box 23-141, Taipei 10617, Taiwan \\ ${ }^{9}$ Research School of Astronomy and Astrophysics, Mount Stromlo Observatory, Australia \\ ${ }^{10}$ Sydney Institute for Astronomy, School of Physics, The University of Sydney, NSW 2006, Australia \\ ${ }^{11}$ Astronomy \& Astrophysics Department, Pennsylvania State University, University Park, PA 16802, USA \\ 12 Korea Astronomy and Space Science Institute, 61-1, Whaam-dong, Yuseong-gu, Daejeon 305-348, Republic of Korea \\ ${ }^{13}$ National Astronomical Observatory of Japan, 2-21-1, Osawa, Mitaka, Tokyo 181-8588, Japan \\ ${ }^{14}$ Institute of Astronomy, The University of Tokyo, 2-21-1 Osawa, Mitaka, Tokyo 181-0015, Japan \\ ${ }^{15}$ Department of Astronomy, University of Massachusetts, Amherst, MA 01003, USA \\ ${ }^{16}$ Instituto Nacional de Astrofisica, Optica y Electronica, Tonantzintla, Aptdo. Postal 51 y 216, 72000 Puebla, Pue., Mexico \\ Received 2010 October 5; accepted 2011 February 25; published 2011 April 6
}

\begin{abstract}
We report new mid-infrared (MIR) observations of the remarkable object IRAS 15099-5856 using the space telescopes AKARI and Spitzer, which demonstrate the presence of prominent crystalline silicate emission in this bright source. IRAS 15099-5856 has a complex morphology with a bright central compact source (IRS1) surrounded by knots, spurs, and several extended $\left(\sim 4^{\prime}\right)$ arc-like filaments. The source is seen only at $\geqslant 10 \mu \mathrm{m}$. The Spitzer mid-infrared spectrum of IRS1 shows prominent emission features from Mg-rich crystalline silicates, strong [Ne II] $12.81 \mu \mathrm{m}$, and several other faint ionic lines. We model the MIR spectrum as thermal emission from dust and compare with the Herbig Be star HD 100546 and the luminous blue variable R71, which show very similar MIR spectra. Molecular line observations reveal two molecular clouds around the source, but no associated dense molecular cores. We suggest that IRS1 is heated by UV radiation from the adjacent O star Muzzio 10 and that its crystalline silicates most likely originated in a mass outflow from the progenitor of the supernova remnant (SNR) MSH 15-52. IRS1, which is embedded in the SNR, could have been shielded from the SN blast wave if the progenitor was in a close binary system with Muzzio 10. If MSH 15-52 is a remnant of Type $\mathrm{Ib} / \mathrm{c}$ supernova

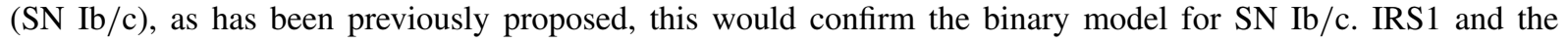
associated structures may be the relics of massive star death, as shaped by the supernova explosion, the pulsar wind, and the intense ionizing radiation of the embedded $\mathrm{O}$ star.
\end{abstract}

Key words: circumstellar matter - infrared: stars - ISM: individual objects (MSH 15-52) - ISM: supernova remnants - stars: individual (IRAS 15099-5856)

Online-only material: color figures

\section{INTRODUCTION}

Space infrared observations have revealed distinct spectral features due to crystalline silicates in diverse objects such as asymptotic giant branch (AGB) stars, planetary nebulae, Herbig Ae/Be stars, comets, and ultraluminous infrared galaxies (Henning 2010). Since silicate dust grains in the interstellar medium are essentially amorphous (Kemper et al. 2004), crystalline silicates must form in circumstellar disks and/or outflows of evolved stars or young stellar objects (YSOs). However, neither the formation process of the crystalline dust nor its relation to the central stellar source is understood.

In this paper, we report the discovery of prominent crystalline silicate emission in IRAS 15099-5856, a bright, mid-infrared compact source previously detected by Infrared Astronomical Satellite (IRAS) and Midcourse Space Experiment (MSX). The source is located close to the pulsar B1509-58 in the supernova remnant (SNR) MSH 15-52 (G320.4-1.2), a young SNR of complex morphology at a distance of $5.2 \pm 1.4 \mathrm{kpc}$ (Arendt 1991; Gaensler et al. 1999; see Section 5). Arendt (1991) concluded that IRAS 15099-5856 is heated either by hot plasma associated with the SNR or by the nearby O star Muzzio 10 (Muzzio 1979), which is at a distance $\sim 4 \mathrm{kpc}$ (M. S. Bessell et al. 2011, in preparation). We show that the latter explanation is most likely correct and that the source is probably associated with the progenitor of the SNR.

\section{AKARI IMAGES AND SPECTRAL ENERGY DISTRIBUTION}

We observed IRAS 15099-5856 using the Infrared Camera (IRC) aboard AKARI on 2007 February 21-22 (ID: 1400761.1 and 1400762.1). The IRC is equipped with three waveband 

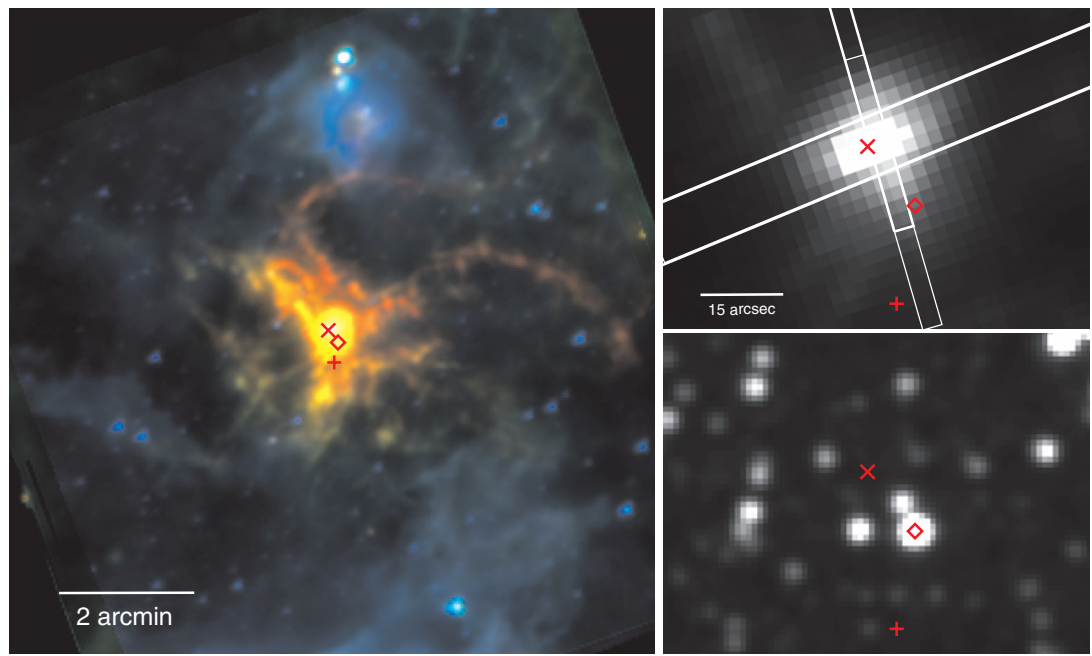

Figure 1. Left: three-color image of IRAS $15099-5856$ produced from AKARI S11 (blue), L15 (green), and L24 (red) images. North is up and east is to the left. The brightness is adjusted to show the faint emission features, which saturated the central part. The cross marks the peak position of the central compact source (IRS1) at $15 \mu \mathrm{m},\left(\alpha_{2000}, \delta_{2000}\right)=\left(15^{\mathrm{h}} 13^{\mathrm{m}} 56^{\mathrm{s}} 32,-59^{\circ} 07^{\prime} 40^{\prime \prime} 9\right)$. The plus and diamond signs mark the positions of the pulsar B1509-58 (Gaensler et al. 2002) and the O star Muzzio 10 (2MASS 15135520-5907516), respectively. The bright star embedded in the diffuse blue emission in the south is the Wolf-Rayet star WR65, while the other bright star of similar morphology in the north is not previously known. Right: magnified view of the central area in L15 band (upper panel) and the corresponding 2MASS image (lower panel). The Spitzer IRS slit positions are shown by rectangular boxes in the upper panel; the large $\left(148^{\prime \prime} \times 10^{\prime \prime} 7\right)$ solid one representing the LL band slit position and two superposed small $\left(500^{\prime \prime} 4 \times 33^{\prime \prime} 7\right)$ solid ones representing the two nodded SL band slit positions.

(A color version of this figure is available in the online journal.)

channels covering the 2.6-26 $\mu \mathrm{m}$ wavelength range with a $10^{\prime} \times$ $10^{\prime}$ field of view (FOV; Onaka et al. 2007). We used all three channels in IRC02 mode, which gave six band images, N3, N4, S7, S11, L15, and L24, with central wavelengths of 3.2, 4.1, 7, 11,15 , and $24 \mu \mathrm{m}$, respectively, and angular resolutions ranging from 4.'2 (N3) to 6." 8 (L24). The data were processed using the standard IRC Imaging Data Reduction Pipeline version 070104. The positional uncertainty $(1 \sigma)$ is $\leqslant 0$ ' 15 for N3- and N4-band images and $\leqslant 0$ '.32 for the longer-wavelength images except the L24-band image, which has a relatively large uncertainty $\left(\sim 1^{\prime \prime}\right)$ because the astrometric solution could not be obtained by matching with the coordinates of Two Micron All Sky Survey (2MASS) stars in the pipeline.

The AKARI observations reveal that IRAS $15099-5856$ is a large, MIR source with spectacular morphology (Figure 1). It is composed of a bright central compact source (hereafter IRS1), a surrounding halo of $\sim 1^{\prime}$ radius with knots and spurs embedded in it, and several extended $\left(\sim 4^{\prime}\right)$, knotty arc-like filaments. The extent of the whole structure is about $10^{\prime}$ and its total flux is $\sim 12 \mathrm{Jy}$ at $15 \mu \mathrm{m}, 40 \%$ of which is from IRS1. The $15 \mu \mathrm{m}$ image in the right frame shows that IRS1 has a brightest central part extended along the east-west direction and some diffuse emission around it. There is no 2MASS counterpart to IRS1. The source is not seen in the IRC images at wavelengths $\lambda<10 \mu \mathrm{m}$.

Figure 2 shows the one-dimensional 24, 15, and $11 \mu \mathrm{m}$ intensity profiles of IRS1 along the east-west $\left(\right.$ P.A. $=110^{\circ}$ ) and north-south directions, which are the two orthogonal directions of the AKARI IRC. We have subtracted a constant background brightness from each profile and normalized it by its maximum brightness: background brightness of about 150 , 60 , and $30 \mathrm{MJy} \mathrm{sr}^{-1}$ and maximum brightness of 3700,1110 , and $30 \mathrm{MJy} \mathrm{sr}^{-1}$ for L24, L15, and S11 profiles, respectively. For comparison, we also show the point-spread function (PSF) of the AKARI IRC. The 15 and $24 \mu \mathrm{m}$ profiles clearly show that IRS1 is composed of a compact bright central part (hereafter, the core) and an extended envelope of $\sim 30^{\prime \prime}$ size. The envelope appears as a plateau in the north-south profile, which suggests that it is a separate component from the core. The core is almost symmetrically elongated along the east-west direction, while it is barely resolved in the north-south direction. The average full-width at half-maximum (FWHM) of the core at $15 / 24 \mu \mathrm{m}$ after deconvolving the PSF is 9'.6 $\left(00^{\prime} 1\right) \times 5^{\prime \prime} .1\left(1^{\prime \prime} .1\right)$ or $0.19 d_{4} \mathrm{pc} \times 0.10 d_{4} \mathrm{pc}$, where $d_{4}$ is the distance in units of $4 \mathrm{kpc}$. (We normalize the distance by $4 \mathrm{kpc}$, which is the distance to the Cir OB1 association in this area; see Section 5.) At $11 \mu \mathrm{m}$, the core has a comparable size (9.7) along the east-west direction, but its center is slightly (1".5) shifted to the west. Along the north-south direction, it appears somewhat more extended (6."4), but this could be due to the contribution from nearby faint stellar sources (see Figure 1). The local peak at $-13^{\prime \prime}$ in the $11 \mu \mathrm{m}$ north-south profile is due to Muzzio 10 .

Table 1 summarizes the observed fluxes of IRAS 15099-5856, where we also list the corresponding "colorcorrected" fluxes, i.e., the fluxes corrected for the spectral slope over the passbands. The color correction has been made by assuming modified blackbody emission at $75 \mathrm{~K}$ that fits the overall shape of the spectral energy distribution (SED). But as we show in Section 3, the MIR emission at $\leqslant 35 \mu \mathrm{m}$ is dominated by spectral bumps from crystalline and metallic dusts, so that the color-corrected fluxes of those bands should be used with caution. The AKARI IRC fluxes are derived by summing the fluxes within a circle of $25^{\prime \prime}$ radius centered on the brightness peak; the contribution from the $\mathrm{O}$ star Muzzio 10 is excluded. The IRAS and MSX fluxes are from the point-source catalogs and they roughly agree with the $A K A R I$ IRC fluxes. At wavelengths shorter than the $A K A R I$ IRC bands, we identified several point sources of 17th-20th mag around IRS1 in the $K_{s}$ image of the source obtained with the Magellan $6.5 \mathrm{~m}$ telescope (Kaplan \& Moon 2006). They are separated from the $15 \mu \mathrm{m}$ peak position by $\geqslant 1$.' 1 , which is greater than the $3 \sigma$ positional uncertainty of the $15 \mu \mathrm{m}$ image. We use the $3 \sigma$ upper limit (20th mag or $0.007 \mathrm{mJy}$ ) of the Magellan observations as an upper limit at $2.15 \mu \mathrm{m}$. At longer wavelengths, we identified the source in the AKARI FIS (Far-Infrared Surveyor) catalog at 65 and $90 \mu \mathrm{m}$ but not at 140 or $160 \mu \mathrm{m}$. With a spatial resolution of $37^{\prime \prime}-61^{\prime \prime}$, the FIS does not resolve IRS1. We also carried out $1.1 \mathrm{~mm}$ 

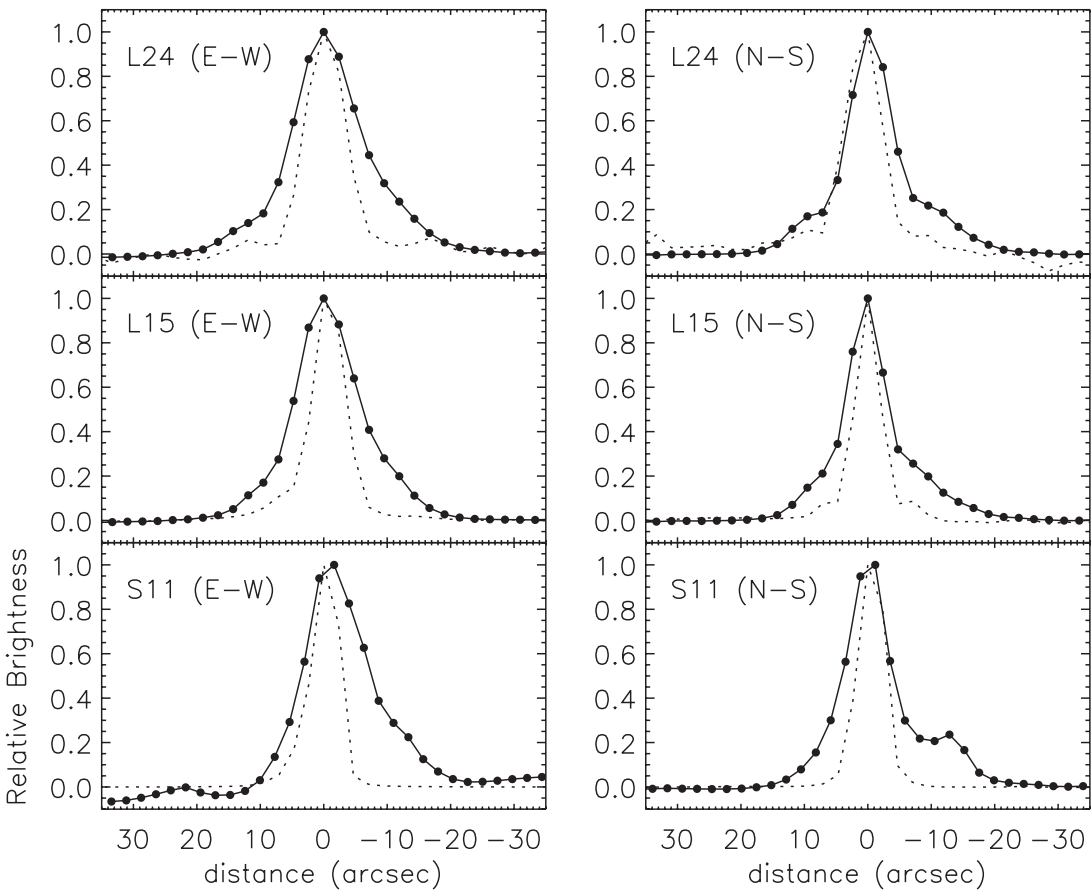

Figure 2. One-dimensional, normalized intensity profiles of the central compact source IRS1 at 24, 15 , and $11 \mu \mathrm{m}$ (solid lines). The left panels show the profiles along the east-west direction $\left(\mathrm{P} . \mathrm{A} .=110^{\circ}\right.$ ), while the right panels show the profiles perpendicular to it. The $(0,0)$ position represents the peak position at $15 \mu \mathrm{m}$ and the filled circles represent the intensities at individual pixels. The $24 \mu \mathrm{m}$ image has a relatively large uncertainty in astrometry, and therefore the profile is shifted to match the peak position at $15 \mu \mathrm{m}$. The pixel sizes are 2".34-2".38. The dotted lines show the profiles of a point source in the same images.

Table 1

Flux of IRAS 15099-5856 IRS1

\begin{tabular}{lccc}
\hline \hline \multirow{2}{*}{ Telescope } & & \multicolumn{2}{c}{ Flux } \\
\cline { 2 - 4 } & $\begin{array}{c}\text { Wavelength } \\
(\mu \mathrm{m})\end{array}$ & $\begin{array}{c}\text { Observed } \\
(\mathrm{Jy})\end{array}$ & $\begin{array}{c}\text { Color Corrected } \\
(\mathrm{Jy})\end{array}$ \\
\hline Magellan & 2.15 & $\leqslant 7 \times 10^{-6}$ & $\ldots$ \\
MSX & 8.28 & $\leqslant 0.101^{\mathrm{a}}$ & $\ldots$ \\
& 14.65 & $1.43(0.10)$ & $1.20(0.08)$ \\
AKARI IRC & 21.3 & $12.88(0.77)$ & $12.50(0.75)$ \\
& 7 & $\leqslant 0.0013$ & $\ldots$ \\
& 11 & $0.116(0.003)$ & $0.076(0.002)$ \\
AKARI FIS & 15 & $5.14(0.15)$ & $1.75(0.02)$ \\
& 24 & $21.4(1.0)$ & $23.5(1.1)$ \\
& 65 & $27.9(4.8)$ & $26.6(4.6)$ \\
IRAS & 90 & $17.2(2.5)$ & $11.0(1.6)$ \\
& 140 & $\leqslant 11^{\mathrm{b}}$ & $\ldots$ \\
& 160 & $\leqslant 25^{\mathrm{b}}$ & $\ldots$ \\
AzTEC & 12 & $\leqslant 1.89$ & $\ldots$ \\
& 25 & $19.9(2.0)$ & $23.8(2.4)$ \\
& 60 & $37.0(4.1)$ & $31.5(3.5)$ \\
& 100 & $\leqslant 138^{\mathrm{c}}$ & $\ldots$ \\
\hline
\end{tabular}

Notes. Magellan, AKARI IRC, and AzTEC fluxes are from this work. For the non-detections, the listed fluxes are $2 \sigma$ upper limits. The other fluxes are from the point-source catalogs.

${ }^{a} M S X$ flux limit with quality_flag=1 (Egan et al. 2003).

${ }^{\mathrm{b}}$ Detection limits according to the FIS bright-source catalog version 1 .

c Upper limits from the IRAS point-source catalog version 2.0, which are nominally $3 \sigma$ values.

continuum observations using the 144 element bolometer array AzTEC (Wilson et al. 2008) on the $10 \mathrm{~m}$ telescope Atacama Submillimeter Telescope Experiment (ASTE; Ezawa et al. 2004) in 2008 October. The beam size and FOV were $28^{\prime \prime}$ and 7.5, respectively. We obtained 11 maps of a $25^{\prime} \times 25^{\prime}$ area in a raster

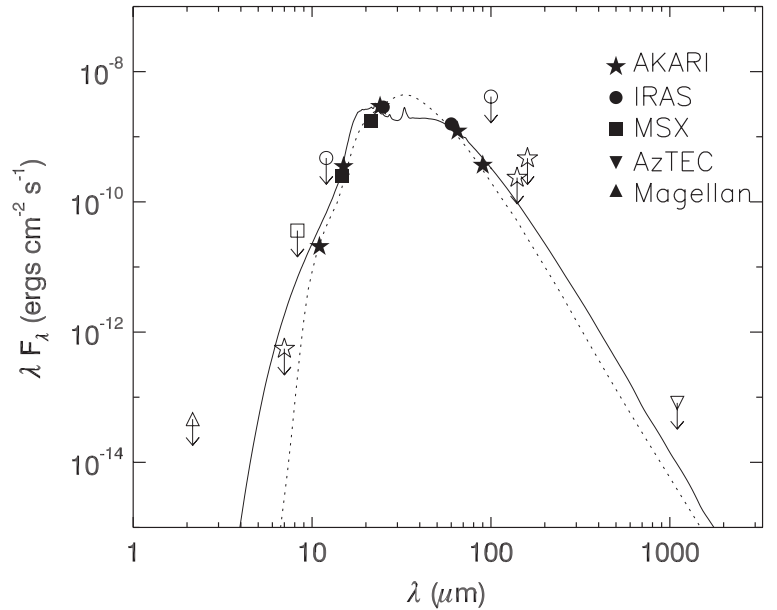

Figure 3. Spectral energy distribution of IRS1 of IRAS 15099-5856. The open symbols represent upper limits. The fluxes are color corrected (see Table 1). The dotted line is a model SED of thermal dust emission at $79 \mathrm{~K}$, whereas the solid line is a model SED resulting from a fit to the Spitzer IRS spectrum (Model 1 in Table 2; see Section 3).

scan at $90^{\prime \prime}$ per second and coadded the individual maps to obtain the final map with a pixel size of $6^{\prime \prime}$. The total on-source observing time was $3 \mathrm{hr}$ and the pixel ${ }^{-1}$ integration time was $10 \mathrm{~s}$. The absolute flux calibration was done by observing Uranus. IRAS 15099-5856 was not detected with an upper limit of 30 mJy $(2 \sigma)$.

Figure 3 shows the SED of IRS1. The flux of IRS1 increases steeply from short wavelengths to $25 \mu \mathrm{m}$ and remains flat at $\sim 30 \mathrm{Jy}$ to $90 \mu \mathrm{m}$. The MIR $(11-25 \mu \mathrm{m})$ spectral index is $\alpha=-7.7\left(F_{\nu} \propto v^{\alpha}\right)$. The SED can be approximately fitted by emission from $2.4 \times 10^{-3} d_{4}^{2} M_{\odot}$ of dust at $79 \mathrm{~K}$ using Draine's carbonaceous-silicate opacity model for interstellar dust with 
$R_{V}=5.5$ (Draine 2003). The fit, however, is not good, with a large reduced $\chi^{2}(=9.4)$. This is because the flux at $\lesssim 35 \mu \mathrm{m}$ is mainly from dust of circumstellar origin with several distinct opacities (Section 3). In Section 3, we discuss the model based on the Spitzer IRS spectra, where we show that the dust mass is about four times larger because the dust temperature is lower. The total IR luminosity of IRS1 is $L_{\mathrm{IRS} 1} \approx 2.0 \times 10^{3} d_{4}^{2} L_{\odot}$.

\section{SPITZER IRS SPECTRUM AND CRYSTALLINE SILICATE EMISSION}

We carried out Spitzer IRS staring observations of IRS1 of IRAS 15099-5856 with the two low-resolution modules; the short-low (SL) module $(5.2-14.5 \mu \mathrm{m})$ and the long-low (LL) module $(14.0-38.0 \mu \mathrm{m})$. The observations were done on 2008 October 3 (ID: 26318080 and 26318336). We followed the standard IRS staring mode, where the spectra were obtained by placing the source at two locations for each slit, located $1 / 3$ and $2 / 3$ of the way along the slits' length (Figure 1). At each location, two to three cycles of different ramp durations (6-60 s) were carried out. We use the coadded two-dimensional spectra provided by the Spitzer Science Center (SSC), which are averaged basic calibration data (BCD) FITS files processed by the pipeline version 17. For the data analysis, we use the Spitzer IRS Custom Extraction software v2.2 provided by the SSC. The IRS1 is extended, so that the flux that misses the slit, i.e., "slit loss," needs to be properly accounted for the flux calibration (see the SSC IRS calibration web page on extended sources ${ }^{17}$ ). We derive the slit-loss correction factor (SLCF) at 11, 15, and $24 \mu \mathrm{m}$ by calculating the fraction of the flux within the SL and LL slits on the AKARI images using the PSF in Sloan et al. (2003b). The SLCFs over the entire SL and LL bands are then obtained by interpolating/extrapolating these data points.

The Spitzer IRS spectrum of IRS1 (Figure 4) increases steeply from $\lesssim 0.1 \mathrm{Jy}$ at $\lesssim 13 \mu \mathrm{m}$ to $20-30 \mathrm{Jy}$ at $\geqslant 20 \mu \mathrm{m}$, which is consistent with the broadband SED. The spectrum shows two broad, strong peaks at 23 and $34 \mu \mathrm{m}$ and also weak features at 20 and $27 \mu \mathrm{m}$. Such spectral features are characteristic of Mg-rich crystalline silicates, e.g., forsterite $\left(\mathrm{Mg}_{2} \mathrm{SiO}_{4}\right)$, and have been observed in AGB stars, YSOs, and comets (Henning 2010). Indeed, the $\geqslant 15 \mu \mathrm{m}$ part of the spectrum is very similar to those of YSOs such as HD 100546 or RECX 5 (Figure 4). HD 100546 is the Herbig Be star (B9.5) that has an exceptionally large crystallinity in the Infrared Space Observatory (ISO) sample of Herbig Ae/Be stars (Malfait et al. 1998; Meeus et al. 2001). RECX 5 is an M4-type pre-main-sequence star in the $\eta$ Chamaeleontis cluster and shows a very similar spectrum to HD 100546 (Bouwman et al. 2005). The spectrum is also quite similar to that of luminous blue variables (LBVs) such as R71 (HD 269009) in the Large Magellanic Cloud (LMC) (Figure 4). R71 is a well-studied LBV with $T_{*}=15,000 \mathrm{~K}$ and $L=5 \times 10^{5} L_{\odot}$ (Boyer et al. 2010). Its $I S O$ and Spitzer IRS spectra show a broad bump steeply rising from $15 \mu \mathrm{m}$ and flattening around $18 \mu \mathrm{m}$, which is very similar to that of IRS1 (Voors et al. 1999; Morris et al. 2008). This bump had been attributed to the metal oxide FeO (Voors et al. 1999). It also shows the weak bumps due to Mg-rich crystalline silicates. The closely matching spectral features suggest that the grain composition should be similar in these sources. A noticeable difference, however, is the absence of crystalline/amorphous silicate emission at $10-11 \mu \mathrm{m}$ in IRAS $15099-5856$, which is distinct in the other sources. This indicates that IRAS

\footnotetext{
17 http://ssc.spitzer.caltech.edu/irs/calib/extended_sources/
}

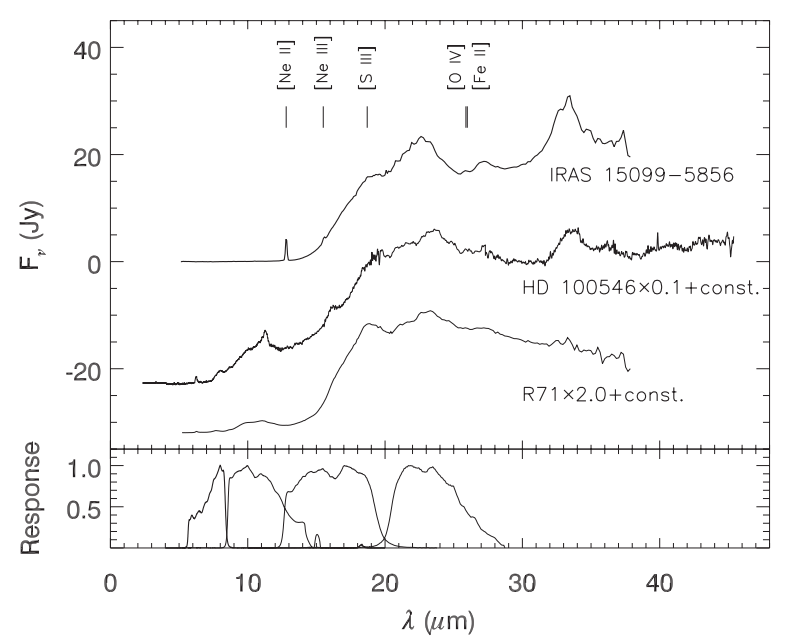

Figure 4. Spitzer IRS spectrum of IRAS 15099-5856. Wavelengths of the identified ionic lines are marked. The $\geqslant 15 \mu \mathrm{m}$ part of the IRAS $15099-5856$ spectrum is very similar to the spectrum of the Herbig Be (B9.5e) star HD 100546 (Malfait et al. 1998) and the LBV R71 (HD 269006) in the LMC (Voors et al. 1999; Morris et al. 2008), which are shown for comparison. The HD 100546 spectrum is from the ISO Short Wavelength Spectrometer spectral atlas of Sloan et al. (2003a), while the R71 spectrum is from the Spitzer Heritage Archive. The bottom panel shows the relative responses of the AKARI S7, S11, L15, and L24 imaging bands.

15099-5856 is missing the warm (several hundred K) silicate dust that the other sources have. Before closing the comparison to other MIR spectra with similar features, we note that dust emission has been recently detected toward several oxygen-rich SNRs, e.g., Cas A, G292.0+1.8, and the LMC SNR N132D (Rho et al. 2008; Ghavamian et al. 2009; Tappe et al. 2006). The $17-22 \mu \mathrm{m}$ features in the spectra of these objects have been attributed to either silicate dust newly formed in supernova ejecta or swept-up polycyclic aromatic hydrocarbons. They, however, do not show crystalline silicate features.

Several ionic lines are present in IRAS 15099-5856: strong [Ne II] $12.81 \mu \mathrm{m}$ and weak [Ne III] $15.56 \mu \mathrm{m}$, [S III] $18.71 \mu \mathrm{m}$, and [O IV] $25.89 /[\mathrm{Fe}$ II] $25.99 \mu \mathrm{m}$. The central velocity of the [Ne II] line is $-160 \pm 560 \mathrm{~km} \mathrm{~s}^{-1}$, which indicates that IRAS 15099-5856 is not at a cosmological distance. The flux of the [Ne II] $12.81 \mu \mathrm{m}$ line is $\sim 1.2 \times 10^{-11} \mathrm{erg} \mathrm{cm}^{-2} \mathrm{~s}^{-1}$. The ratio $[\mathrm{Ne} \mathrm{III}] /[\mathrm{Ne} \mathrm{II}] \sim 0.14$, although this is uncertain since the lines appear in different, almost orthogonal, slits. The spatial extent (FWHM) of the [Ne II] line-emitting region along the north-south-oriented SL slit is $5^{\prime \prime}$, while that of the dustcontinuum-emitting region along the east-west-oriented LL slit is $12^{\prime \prime}-13^{\prime \prime}$. Considering the uncertainties in the size measurement with the slit spectrograph, e.g., slight misalignment of the slit from the peak position, we consider that these results are consistent with the size of IRS1 determined from the AKARI IRC image in Section 2.

We model the IRS spectrum as thermal emission from several independent dust components. We do not carry out a detailed analysis considering all possible dust species, which is beyond the scope of this paper. We instead determine the minimum number of dust species required to explain the observed IRS spectra and derive their characteristic parameters. The IRS spectrum in Figure 4 requires at least three dust components. First, the narrow intensity peaks at 22.6, 27.2, and $33.5 \mu \mathrm{m}$ clearly indicate a considerable amount of $\mathrm{Mg}$-rich crystalline olivine, i.e., $\mathrm{Mg}_{2 x} \mathrm{Fe}_{2-2 x} \mathrm{SiO}_{4}$ with $x \approx 1$. The peak frequency and the relative strength of the mass absorption coefficient 

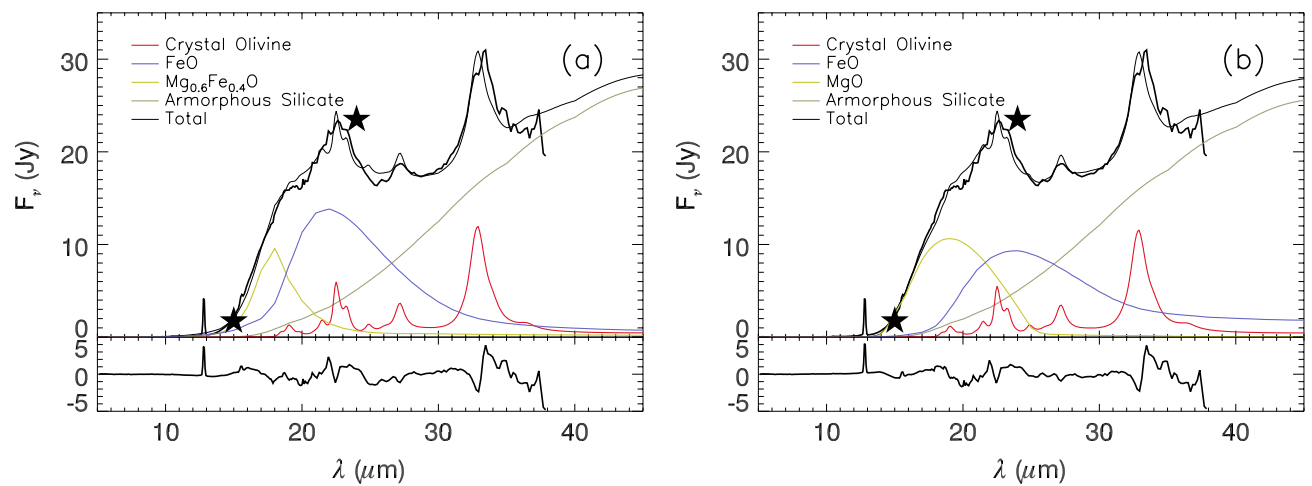

Figure 5. Model fits to the Spitzer IRS spectrum of IRAS 15099-5856 (thick solid lines); (a) Model 1 and (b) Model 2 in Table 2, respectively. The thin solid line in each plot represents a model of thermal emission from several independent dust components, which are shown. The filled star symbols mark the 15 and $24 \mu \mathrm{m}$ AKARI fluxes. The bottom frames show the differences between the model fits and the observed spectrum.

(A color version of this figure is available in the online journal.)

Table 2

Dust Parameters of IRS1 from the Spitzer IRS Spectrum

\begin{tabular}{lccccc}
\hline \hline & \multicolumn{2}{c}{ Model 1} & & \multicolumn{2}{c}{ Model 2} \\
\cline { 2 - 3 } \cline { 5 - 6 } \multicolumn{1}{c}{ Component } & Mass & Temperature & & Mass & Temperature \\
& $\left(10^{-3} d_{4}^{2} M_{\odot}\right)$ & $(\mathrm{K})$ & & $\left(10^{-3} d_{4}^{2} M_{\odot}\right)$ & $(\mathrm{K})$ \\
\hline Crystalline olivine $\left(\mathrm{Mg}_{1.9} \mathrm{Fe}_{0.1} \mathrm{SiO}_{4}\right)$ & $2.3(0.7)$ & $54.8(1.6)$ & & $2.5(0.7)$ & $54.1(1.6)$ \\
$\mathrm{FeO}$ & $0.020(0.003)$ & $146(4)$ & & $0.22(0.08)$ & $89.4(4.9)$ \\
$\mathrm{MgO}$ & $\ldots$ & $\ldots$ & & $0.025(0.006)$ & $116(3)$ \\
$\mathrm{Mg}_{0.6} \mathrm{Fe}_{0.4} \mathrm{O}$ & $0.0069(0.0016)$ & $147(6)$ & & $\ldots$ & $\ldots$ \\
Amorphous silicate & $6.6(0.3)$ & $57.2(0.4)$ & & $6.1(0.2)$ & $57.6(0.4)$ \\
\hline Total & $8.9(1.0)^{\mathrm{a}}$ & $\ldots$ & $8.9(1.0)^{\mathrm{a}}$ & $\ldots$ \\
\hline
\end{tabular}

Notes. ${ }^{a}$ Since the estimates of the mass of the different components are not independent, we give an upper limit on the error in the total mass by simply adding the errors of the individual components.

(hereafter MAC) depend on the $\mathrm{Fe} / \mathrm{Mg}$ ratio, particle shape, particle size, and temperature (Fabian et al. 2001; Koike et al. 2003 , 2006). The strong peaks at the above wavelengths are characteristics of Mg-rich, Fe-poor olivines (see Figure 1 of Koike et al. 2003). We adopt $\mathrm{Mg}_{1.9} \mathrm{Fe}_{0.1} \mathrm{SiO}_{4}$ and use the optical constants of Fabian et al. (2001). We assume spherical dust grains with uniform radii of $0.1 \mu \mathrm{m}$ and calculate their MAC following Bohren \& Huffman (1983). Second, the broad bump steeply rising from $15 \mu \mathrm{m}$ and flattening around $18 \mu \mathrm{m}$ could be attributed to metal oxides $\left(\mathrm{Fe}_{x} \mathrm{Mg}_{1-x} \mathrm{O}, 0 \leqslant x \leqslant 1\right)$. Metal oxides have single-peaked, broad MACs with peak wavelengths between 16 and $22 \mu \mathrm{m}$ depending on the $\mathrm{Fe}$ content (Henning et al. 1995). We use a combination of $\mathrm{FeO}$ and $\mathrm{Mg}_{0.6} \mathrm{Fe}_{0.4} \mathrm{O}$ with optical constants of Henning et al. (1995). The MACs of $0.1 \mu \mathrm{m}$ spherical particles, however, are rather narrow to fit the observed broad bump, and we use the MAC for continuous distribution of ellipsoids for $\mathrm{FeO}$ using a quadratic weighting where near-spherical particle shapes are most probable. Different combinations of metal oxides might fit the spectrum equally well. For example, $\mathrm{MgO}$ has an absorption peak at wavelengths shorter than that of $\mathrm{FeO}$ and can be used instead of $\mathrm{Mg}_{0.6} \mathrm{Fe}_{0.4} \mathrm{O}$ (see below). Finally, we need an additional, cool dust component for the far-IR emission that peaks at about $60 \mu \mathrm{m}$. Since we have only broadband fluxes at $\geqslant 35 \mu \mathrm{m}$, we cannot constrain the composition of this component. We adopt amorphous silicate and use the optical constants of cool silicate grains derived by Suh (1999).

With the above four dust components, i.e., crystalline olivine, $\mathrm{FeO}, \mathrm{Mg}_{0.6} \mathrm{Fe}_{0.4} \mathrm{O}$, and amorphous silicate, we fit the Spitzer IRS spectrum using MPFIT, which is a least-squares fitting tool based on the Levenberg-Marquardt algorithm (Markwardt 2009). In the fit, we leave the temperatures and the masses of individual components free with reasonable lower and upper boundaries. In order to constrain the far-IR part of the spectrum, we use the AKARI 65 and $90 \mu \mathrm{m}$ fluxes. The best-fit masses and temperatures obtained by least-squares fitting are given in Table 2, where we also show the results when $\mathrm{MgO}$ is used instead of $\mathrm{Mg}_{0.6} \mathrm{Fe}_{0.4} \mathrm{O}$ (Model 2). The resulting model spectrum fits the observed IRS spectrum and also the far-IR broadband fluxes reasonably well (Figures 2 and 5). The other model (Model 2) can fit the spectrum equally well (Figure 5). We could not exactly reproduce the observed spectrum because of the simplicity of the adopted dust model and because the dust components are unlikely to be isothermal. The total dust mass is $9 \times 10^{-3} d_{4}^{2} M_{\odot}$, which is considerably greater than the estimate in Section 2 because of the lower temperature of the silicate dust components. Most of the mass comes from amorphous silicate, the cool dust component for the far-IR emission, while crystalline silicates contribute about $25 \%$. The temperatures and therefore the masses of these two dust components vary little between the two models. However, the parameters of the metal oxide components vary significantly, as can be seen from Table 2, so they are not reliable. The temperature of crystalline silicate $(55 \mathrm{~K})$ is much lower than that $(210 \mathrm{~K})$ in HD 100546 (Malfait et al. 1998). The corresponding temperature in R71 is not available, but the temperature of the dust responsible for most of the emission at 10-100 $\mu \mathrm{m}$ in R71 has been estimated to be $120 \mathrm{~K}$ (Boyer et al. 2010). In R71, it was noted that an additional warm dust at $300 \mathrm{~K}$ is needed to explain the bump near $10 \mu \mathrm{m}$ (Voors et al. 1999). 


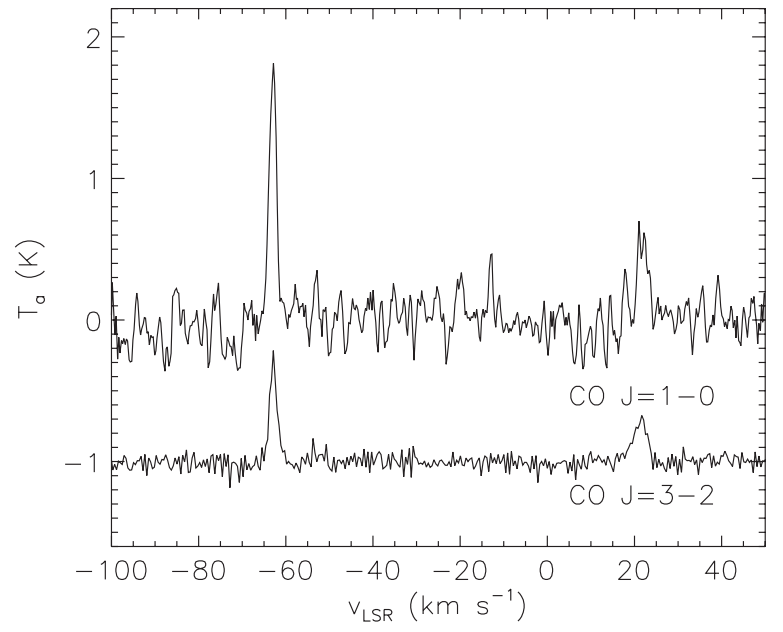

Figure 6. CO $J=1-0$ and $J=3-2$ line profiles toward IRS1. The former is obtained by the $22 \mathrm{~m}$ Mopra telescope (FWHM $=33^{\prime \prime}$ ), while the latter is obtained by the $10 \mathrm{~m}$ ASTE telescope $\left(\mathrm{FWHM}=22^{\prime \prime}\right)$.

\section{MOLECULAR EMISSION LINES AND OTHER WAVEBANDS}

We carried out molecular line observations of IRAS 15099-5856 using the $22 \mathrm{~m}$ telescope at the Mopra Observatory and ASTE. With Mopra, we mapped a $6^{\prime} \times 6^{\prime}$ area surrounding IRAS $15099-5856$ in the $\mathrm{CO} J=1-0$ line at $115.27 \mathrm{GHz}$ $\left(\mathrm{FWHM}=33^{\prime \prime}\right)$ on 2008 June 28 and July 13. With ASTE, we made five-point cross observations at $20^{\prime \prime}$ spacing in the $\mathrm{CO}$ $J=3-2$ line at $345.79\left(\mathrm{FWHM}=22^{\prime \prime}\right)$ from 2008 May 31 to 2008 June 6 . We detected molecular gas at two LSR velocities, $-62 \mathrm{~km} \mathrm{~s}^{-1}$ and $+22 \mathrm{~km} \mathrm{~s}^{-1}$ surrounding IRAS 15099-5856 (Figures 6 and 7). Neither component peaks at the position of IRS1. Instead, the $-62 \mathrm{~km} \mathrm{~s}^{-1}$ component shows a cavity at the position of IRS1, while the $+22 \mathrm{~km} \mathrm{~s}^{-1}$ component shows no obvious spatial correlation. The two clouds have a $\mathrm{CO}(J=3-2) /(J=1-0)$ ratio $\sim 0.5$, which is typical for giant molecular clouds.

We have also searched the $1.4 \mathrm{GHz}$ continuum data of this area obtained from the Australian Compact Telescope Array (ATCA; Gaensler et al. 1999) and a deep (250 ks) Chandra $\mathrm{X}$-ray archival observation, but could not identify a counterpart. The upper limit $(5 \sigma)$ on the $1.4 \mathrm{GHz}$ continuum flux is $\sim 10 \mathrm{mJy}$.

\section{NATURE OF IRAS 15099-5856}

The strong crystalline silicate emission indicates that the dust in IRS1 is circumstellar or perhaps of supernova origin. The steeply rising MIR spectrum, the low-velocity [Ne II] $12.8 \mu \mathrm{m}$ line, and the large extinction through the Galactic plane in this direction almost rule out an extragalactic origin for IRAS 15099-5856. The derived dust mass (Table 2) and the spatial extent $\left(0.19 \times 0.10 d_{4}\right.$ pc $)$ imply a small selfextinction ( $\lesssim 0.1 \mathrm{mag}$ at $K$ band); together with the absence of an embedded star in images of IRAS 15099-5856, this rules out the possibility that it is an extended dust clump powered by an embedded star. It is unlikely that several highly obscured stellar sources form IRS1, since the temperature (55-57 K) of the major dust components is less than expected for such circumstellar dust. Instead, the [Ne II] and [Ne III] emission lines and the IR luminosity suggest that it could be powered by a nearby hot, luminous star. According to stellar atmosphere models, the ratio $[\mathrm{Ne}$ III $] /[\mathrm{Ne}$ II $] \sim 0.14$ implies a stellar temperature of 30,000-40,000 K (Morisset et al. 2004). The observed [Ne II] luminosity implies a Lyman continuum photon luminosity $S_{\text {IRS1 }}=1.2 \times 10^{47} d_{4}^{2} \mathrm{~s}^{-1}$ incident upon IRS1 (Ho \& Keto 2007).

The nearby $\mathrm{O}$ star Muzzio 10 can provide both the required luminosity and the ionizing photons. Based on new optical spectra, M. S. Bessell et al. (2011, in preparation) concluded that its spectral type is $\mathrm{O} 4.5 \mathrm{III}(\mathrm{fp})$ and it is probably a member of the Cir OB1 association at $\sim 4 \mathrm{kpc}$. We adopt $T_{*}=40,500 \mathrm{~K}$, $L_{*}=5.8 \times 10^{5} L_{\odot}$, and $S_{*}=3.2 \times 10^{49} \mathrm{~s}^{-1}$ for Muzzio 10, the parameters of an O4.5III star (Martins et al. 2005). Note that the stellar temperature is consistent with that needed to provide the observed $[\mathrm{Ne} \mathrm{III}] /[\mathrm{Ne} \mathrm{II}]$ line ratio. The projected distance of IRS1 from Muzzio 10 is $13^{\prime \prime} .7$, or $0.27 d_{4} \mathrm{pc}$, comparable to the size of IRS1. At $4 \mathrm{kpc}, L_{\mathrm{IRS} 1} / L_{*}=3.4 \times$ $10^{-3} d_{4}^{2}$ and $S_{\text {IRS } 1} / S_{*}=3.8 \times 10^{-3} d_{4}^{2}$, which are in excellent agreement. If the projected surface area of IRS 1 as seen from Muzzio 10 is the same as that seen from Earth, then they are separated by $0.6 \mathrm{pc}$. The expected temperature of amorphous silicate grains of radius $0.1 \mu \mathrm{m}$ is $70 \mathrm{~K}$ (Tielens 2005), but the mean temperature of dust in a cloud with a large UV-optical depth will be lower. The temperature of $57 \mathrm{~K}$ inferred in Section 3 (Table 2) therefore is consistent with our expectation. For metal oxide dust, however, the expected temperature is considerably lower than the derived temperature $(150 \mathrm{~K})$ in
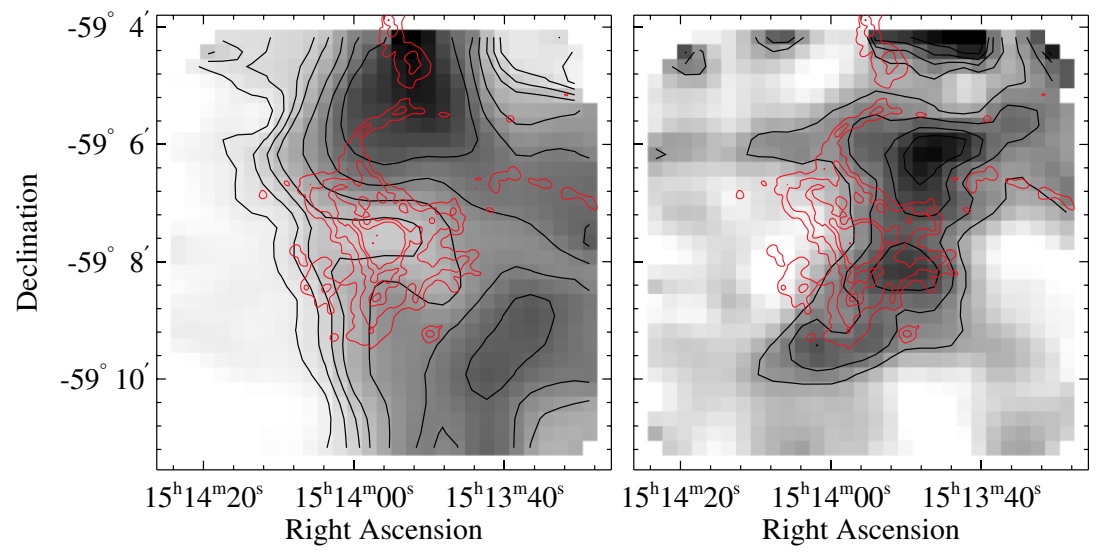

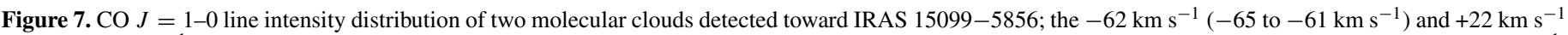

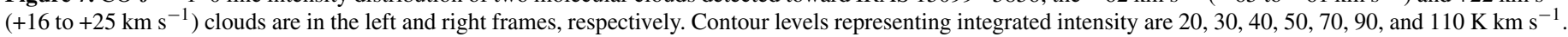
The gray-scale plot distinguishes hills from valleys. The red contours show the $24 \mu \mathrm{m}$ brightness of IRAS $15099-5856$.

(A color version of this figure is available in the online journal.) 
Section 3. This, however, could be due to our assumed dust composition, e.g., $\mathrm{MgO}$ instead of $\mathrm{Mg}_{0.6} \mathrm{Fe}_{0.4} \mathrm{O}$ yields $90-120 \mathrm{~K}$ instead of $150 \mathrm{~K}$ (Table 2). We conclude that the agreement between the predicted and observed $\mathrm{Ne}$ line luminosities, IR luminosities and silicate dust temperatures supports the hypothesis that IRS1 is powered by Muzzio 10, although the high temperature of the small amount of the metal oxide dust remains unexplained. This association implies that IRS1 cannot be too old, since the ionizing radiation from Muzzio 10 would have destroyed it in $\sim 10^{5} \mathrm{yr}$ (Bertoldi \& McKee 1990); it would also be subject to destruction by the stellar wind from Muzzio 10.

What is the origin of the crystalline silicate? It can be produced in both YSOs and evolved stars, since it requires temperatures $\gtrsim 1000 \mathrm{~K}$ to form by thermal annealing (Henning 2010). The dust in YSOs is confined to circumstellar disks and is thus very compact; hence, a YSO origin does not apply to the present source, which is extended. The absence of dense molecular material associated with IRAS $15099-5856$ is consistent with this conclusion. There are no luminous evolved stars within the boundaries of IRS1: Muzzio 10 is very hot and must still be close to the main sequence; it is not in the blue-loop phase of late-stage evolution of massive stars, and its location in the H-R diagram is far from the "S Dor instability strip" that defines the left boundary of the LBVs (Vink 2009). There is a Wolf-Rayet star (WR65; Oskinova \& Hamann 2008) and another possible mass-losing star in the field, but they are relatively distant (4'.3 south of and $4^{\prime} .0$ north of IRS 1 , respectively) and show very different MIR colors (see Figure 1). The only candidate for the source is the SNR MSH 15-52, which is at a comparable distance $(5.2 \pm 1.4 \mathrm{kpc}$; Gaensler et al. 1999), or its progenitor. If we fit the broadband SED of Muzzio 10 from optical to MIR assuming a spectral type of O4.5III, we obtain a distance of $4.5 \mathrm{kpc}$ and an absorbing $\mathrm{H}$ nucleus column density of $9.2 \times 10^{21} \mathrm{~cm}^{-2}$. This column density matches well with that to the SNR determined from X-rays, $9.5 \pm 0.3 \times 10^{21} \mathrm{~cm}^{-2}$ (Gaensler et al. 2002), which supports the association. The crystalline silicates could have been produced either in the late stages of the progenitor's evolution or in the cooling SN ejecta. We favor a progenitor origin for IRS1 in view of its low velocity, $-160 \pm 560 \mathrm{~km} \mathrm{~s}^{-1}$. An origin in the progenitor is consistent with the ubiquity of crystalline silicates in evolved stars. Crystalline silicate emission features have not been detected in the spectra of freshly formed SN dust, although crystalline silicates of core-collapse $\mathrm{SN}$ origin have been discovered in interplanetary dust particles (Messenger et al. 2005). Crystalline silicates are subject to destruction and amorphization by SNR shocks and cosmic rays (Jäger et al. 2003, and references therein), so that they will not survive long in the hostile environment of a young SNR even if they are formed in the SN ejecta.

A progenitor origin for IRS1 has several interesting consequences. First, IRS1 should be embedded in the SNR and be relatively close to the explosion center, because otherwise, considering the large radius ( $23 \mathrm{pc}$ at $4 \mathrm{kpc}$; Gaensler et al. 1999) of the remnant, the dust mass lost by the progenitor would be unacceptably large. This raises the question of how IRS1 could have survived the $\mathrm{SN}$ blast wave, because most dust grains are destroyed by shocks faster than $\sim 200 \mathrm{~km} \mathrm{~s}^{-1}$ (Jones et al. 1996). One possibility would be that the explosion was very asymmetric so that IRS 1 avoided the expanding dense ejecta. Another, more intriguing possibility is that the progenitor and Muzzio 10 formed a close binary system, as first proposed by Gaensler et al. (1999), and that Muzzio 10 shielded IRS1 from the SN blast wave as shown, for example, in numerical simulations of Type Ia SN explosions in binary systems (Marietta et al. 2000). The close proximity of Muzzio 10 and the pulsar B1509-58 gives support to this possibility. Since Muzzio 10 is currently an O4.5III star of $45 M_{\odot}$ (Martins et al. 2005), the progenitor should have had a very large $\left(60-70 M_{\odot}\right)$ initial mass and a separation $\sim 100 R_{\odot}$ from Muzzio 10 (S.-C. Yoon 2011, private communication). The progenitor could have lost most of its mass during Wolf-Rayet phase prior to its explosion as an SN. The shielding scenario for IRS1 implies a distance from the SN: if we assume that the diameter of Muzzio 10 in a binary was comparable to that of an isolated O4.5III star, i.e., $30 R_{\odot}$ (Martins et al. 2005), then it would shield a region $0.2 \mathrm{pc}$ in extent (the observed size of IRS1) from a supernova $100 R_{\odot}$ away if IRS1 were about $0.6 \mathrm{pc}$ from the SN. This is the same as the estimate of the current separation between Muzzio 10 and IRS1 made above. Dust at this distance from the supernova could readily survive the $\mathrm{SN}$, as can be inferred from Draine (2010). If a significant fraction of the mass loss that led to the formation of IRS1 were in a disk of that radius, then the total dust mass of the disk would be about $(2 \pi \times 0.6 \mathrm{pc}) /(0.2 \mathrm{pc})$ times the mass of IRS 1 or about $0.17 M_{\odot}$. For a gas-to-dust ratio of 100 , this implies a total mass of $17 M_{\odot}$, which is large but not unreasonable.

Measurement of the proper motions of the pulsar and Muzzio 10 would be the key test of this binary shielding model. The orbits of the neutron star and its companion after the supernova explosion depend on their separation, masses, and the magnitude of the kick velocity imparted to the neutron star during the explosion (Tauris \& Takens 1998). Since Muzzio 10 is much more massive than the neutron star, its velocity would be almost unchanged by the $\mathrm{SN}$, and as a result its motion should have been nearly perpendicular to the vector from the $\mathrm{SN}$ to IRS1. The motion of the pulsar, on the other hand, could be mainly determined by the magnitude of the kick velocity and so could be in any direction. The available radio data on the pulsar B1509-58, however, do not show an appreciable proper motion (M. Livingstone 2010, private communication). Determination that the progenitor was a member of a binary system, together with the suggestion by Gaensler et al. (1999) that the supernova was of Type Ib/c, would also support the binary model for SN $\mathrm{Ib} / \mathrm{c}$ (e.g., Yoon et al. 2010), although this would require revision of the canonical view that only fast winds are expected in the final evolutionary stages of Type Ib/c progenitors (Chevalier 2005). Above, we commented that the mid-IR spectrum of IRAS 15099-5856 was similar to that of an LBV, which experiences extreme mass loss before its explosion and is associated with SN IIn supernovae (Smith 2010). However, Bessell et al. (2011, in preparation) have obtained optical spectra toward IRS 1 and detected no $\mathrm{H} \alpha$ emission. The absence of emission from hydrogen in the mass lost from the progenitor supports a Type Ib/c origin rather than a Type IIn origin for the supernova.

The nature of the diffuse, extended structures surrounding IRS1 is uncertain. They are not correlated with the X-ray knots or arcs of the pulsar wind nebula (Gaensler et al. 2002). Spitzer IRS spectra of these structures indicate that they are a mixture of dust-continuum dominated material like IRS1 and line-dominated, ionized gas. It is likely that the red/yellow knots, spurs, and filaments in Figure 1 are dominated by dust continuum, while the diffuse green structures are dominated by line emission, in which case the former might be the progenitor's 
circumstellar material while the latter are SN ejecta. In one faint, [Ne II]-emitting gaseous clump, we measured a central velocity $\sim+1000 \mathrm{~km} \mathrm{~s}^{-1}$, consistent with $\mathrm{SN}$ ejecta. The SL and LL slits, which are almost orthogonal to each other, provide only limited spectral and spatial information to reach further conclusions. The spatial relation of IRS1 and the surrounding structures to the SNR as well as to Muzzio 10 needs further study.

IRAS 15099-5856 appears to be the first case in which crystalline silicates have been observed to be associated with an SNR or its progenitor. IRS1 and the associated structures may be the relics of massive star death in a close binary system, as shaped by the supernova, the pulsar wind and the intense ionizing radiation of the embedded $\mathrm{O}$ star. If confirmed by observations of the proper motion of Muzzio 10 and pulsar B1509-58, we have a unique opportunity to study the interior of a young SNR containing crystalline dust and illuminated by a central ionizing source.

This work is in part based on observations with AKARI, a JAXA project with ESA, and with the Spitzer Space Telescope, operated by the Jet Propulsion Laboratory, California Institute of Technology under a contract with NASA. Support for this work was partially provided by NASA through an award issued by JPL/Caltech. We thank Ko Arimatsu for checking the possible effect of optical ghosts in the L15 image. We thank Matthew Bailes, Alex Filippenko, Vicky Kaspi, Maggie Livingstone, Nathan Smith, and Sung-Chul Yoon for their helpful discussions. We also thank the anonymous referee for his/her comments which considerably improved the presentation of the paper. B.C.K. was supported by the National Research Foundation of Korea (NRF) grant funded by the Korea Government (MEST-No. R01-2007-000-20336-0, NRF-2010616-C00020). C.F.M. was supported by NSF grant AST0908553 and by the Groupement d'Intérêt Scientifique (GIS) "Physique des deux infinis (P2I)." The Mopra Telescope and ATCA are both parts of the Australia Telescope supported by the Commonwealth of Australia as a National Facility managed by CSIRO. The University of New South Wales Mopra Spectrometer Digital Filter Bank was provided with support from the Australian Research Council, together with the University of New South Wales, University of Sydney, and Monash University. The ASTE project is operated by Nobeyama Radio Observatory (NRO), a branch of National Astronomical Observatory of Japan (NAOJ), in collaboration with University of Chile, University of Tokyo, Nagoya University, Osaka Prefecture University, Ibaraki University, and Hokkaido University. Observations with ASTE were in part carried out remotely from Japan by using NTT's GEMnet2 and its partner Research and Education networks, which are based on AccessNova collaboration of University of Chile, NTT Laboratories, and NAOJ.

\section{REFERENCES}

Arendt, R. G. 1991, AJ, 101, 2160

Bertoldi, F., \& McKee, C. F. 1990, ApJ, 354, 529
Bohren, C. F., \& Huffman, D. R. 1983, Absorption and Scattering of Light by Small Particles (New York: Wiley)

Bouwman, J., Lawson, W., Feigelson, E. D., Henning, T., Tielens, A. G. G. M., Waters, L. B. F. M., \& Dominik, C. 2005, in Protostars and Planets V, ed. B. Reipurth, D. Jewitt, \& K. Keil (Tucson, AZ: Univ. Arizona Press), 8565

Boyer, M. L., et al. 2010, A\&A, 518, L142

Chevalier, R. A. 2005, ApJ, 619, 839

Draine, B. T. 2003, ARA\&A, 41, 241

Draine, B. T. 2010, arXiv:1003.0474

Egan, M. P., et al. 2003, The Midcourse Space Experiment Point Source Catalog Version 2.3, Technical Report AFRL-VS-TR-2003-1589

Ezawa, H., Kawabe, R., Kohno, K., \& Yamamoto, S. 2004, Proc. SPIE, 5489, 763

Fabian, D., Henning, T., Jäger, C., Mutschke, H., Dorschner, J., \& Wehrhan, O. 2001, A\&A, 378, 228

Gaensler, B. M., Arons, J., Kaspi, V. M., Pivovaroff, M. J., Kawai, N., \& Tamura, K. 2002, ApJ, 569, 878

Gaensler, B. M., Brazier, K. T. S., Manchester, R. N., Johnston, S., \& Green, A. J. 1999, MNRAS, 305, 724

Ghavamian, P., Raymond, J. C., Blair, W. P., Long, K. S., Tappe, A., Park, S., \& Winkler, P. F. 2009, ApJ, 696, 1307

Henning, T. 2010, ARA\&A, 48, 21

Henning, T., Begemann, B., Mutschke, H., \& Dorschner, J. 1995, A\&AS, 112 143

Ho, L. C., \& Keto, E. 2007, ApJ, 658, 314

Jäger, C., Fabian, D., Schrempel, F., Dorschner, J., Henning, T., \& Wesch, W. 2003, A\&A, 401, 57

Jones, A. P., Tielens, A. G. G. M., \& Hollenbach, D. J. 1996, ApJ, 469, 740

Kaplan, D. L., \& Moon, D.-S. 2006, ApJ, 644, 1056

Kemper, F., Vriend, J., \& Tielens, A. G. G. M. 2004, ApJ, 609, 826

Koike, C., Chihara, H., Tsuchiyama, A., Suto, H., Sogawa, H., \& Okuda, H. 2003, A\&A, 399, 1101

Koike, C., et al. 2006, A\&A, 449, 583

Malfait, K., Waelkens, C., Waters, L. B. F. M., Vandenbussche, B., Huygen, E., \& de Graauw, M. S. 1998, A\&A, 332, L25

Marietta, E., Burrows, A., \& Fryxell, B. 2000, ApJS, 128, 615

Markwardt, C. B. 2009, in ASP Conf. Ser. 411, Astronomical Data Analysis Software and Systems XVIII, ed. D. A. Bohlender, D. Durand, \& P. Dowler (San Francisco, CA: ASP), 251

Martins, F., Schaerer, D., \& Hillier, D. J. 2005, A\&A, 436, 1049

Meeus, G., Water, L. B. F. M., Bouwman, J., van den Ancker, M. E., Waelkens, C., \& Malfait, K. 2001, A\&A, 365, 476

Messenger, S., Keller, L. P., \& Lauretta, D. S. 2005, Science, 309, 737

Morisset, C., Schaerer, D., Bouret, J.-C., \& Martins, F. 2004, A\&A, 415, 577

Morris, P., et al., (Spitzer WRRINGS Team) 2008, in IAU Symp. 250, Massive Stars as Cosmic Engines, ed. F. Bresolin, P. A. Crowther, \& J. Puls (Cambridge: Cambridge Univ. Press), 361

Muzzio, J. C. 1979, AJ, 84, 639

Oskinova, L. M., \& Hamann, W.-R. 2008, MNRAS, 390, L78

Onaka, T., et al. 2007, PASJ, 59S, 401

Rho, J., et al. 2008, ApJ, 673, 271

Sloan, G. C., Kraemer, K. E., Price, S. D., \& Shipman, R. F. 2003a, ApJS, 147, 379

Sloan, et al. 2003b, Infrared Spectrograph Technical Report Series, IRSYR03001

Smith, N. 2010, in ASP Conf. Ser. 425, Hot and Cool: Bridging Gaps in Massive Star Evolution, ed. C. Lietherer, P. Bennett, P. Morris, \& J. van Loon (San Francisco, CA: ASP), 63

Suh, K.-W. 1999, MNRAS, 304, 389

Tappe, A., Rho, J., \& Reach, W. T. 2006, ApJ, 653, 267

Tauris, T. M., \& Takens, R. J. 1998, A\&A, 330, 1047

Tielens, A. G. G. M. (ed.) 2005, in The Physics and Chemistry of the Interstellar Medium (Cambridge: Cambridge Univ. Press), 129

Vink, J. S. 2009, arXiv:0905.3338

Voors, R. H. M., Waters, L. B. F. M., Morris, P. W., Trams, N. R., de Koter, A., \& Bouwman, J. 1999, A\&A, 341, L67

Wilson, G. W., et al. 2008, MNRAS, 386, 807

Yoon, S.-C., Woosley, S.-E., \& Langer, N. 2010, ApJ, 725, 940 